\title{
Effects of hydroxyhydroquinone-reduced coffee on vasoreactivity and blood pressure
}

\author{
Ryuji Ochiai $^{1}$, Akiro Chikama ${ }^{1}$, Kiyoshi Kataoka ${ }^{1}$, Ichiro Tokimitsu ${ }^{1}$, Yoshihiro Maekawa ${ }^{2}$, Mitsuru Ohishi ${ }^{2}$, \\ Hiromi Rakugi ${ }^{2}$ and Hiroshi Mikami ${ }^{3}$
}

Recent studies suggest that chlorogenic acids, which are the main components of the polyphenol class in coffee, decrease blood pressure, and that hydroxyhydroquinone (HHQ), which is generated by roasting coffee beans, inhibits the antihypertensive effect of chlorogenic acids in brewed coffee. Here, we examined the vasoreactivity and antihypertensive effects of HHQ-reduced coffee in mild hypertension. The study design was a double blind, randomized, placebo-controlled intervention study, with a 4-week run-in period, followed by an 8-week test beverage ingestion period. The subjects were Japanese men and women with mild hypertension and vascular failure, who were not taking any antihypertensive drugs. During the test beverage ingestion period, the subjects ingested either active or placebo HHQ-reduced coffee (chlorogenic acids per $184 \mathrm{ml}$ of coffee: active, $300 \mathrm{mg}$; and placebo, $0 \mathrm{mg}$ ) daily. Subjects were randomly divided into two groups: active group $(n=9)$ and placebo group $(n=12)$. In the active beverage group, endothelium-dependent, flow-mediated vasodilation impairment was significantly ameliorated and systolic blood pressure was significantly decreased from the baseline, but not in the placebo group. There were no test beverage consumption-related changes in other parameters that may influence blood pressure, such as pulse, cardiac output, body weight or 24-h urine volume. Ingestion of the active beverage significantly decreased urinary isoprostane levels, suggesting a reduced oxidative stress. These findings indicate that HHQ-reduced coffee decreased blood pressure in subjects with mild hypertension. The decreased blood pressure was associated with improved vascular endothelial function.

Hypertension Research (2009) 32, 969-974; doi:10.1038/hr.2009.132; published online 28 August 2009

Keywords: chlorogenic acid; clinical trial; coffee; endothelium; hydroquinones

\section{INTRODUCTION}

Coffee is widely ingested throughout the world and many studies have examined the effects of brewed coffee on various diseases, such as type 2 diabetes, ${ }^{1,2}$ liver dysfunction ${ }^{3,4}$ and Parkinson's disease. ${ }^{5}$ Effects of the caffeine, contained in coffee, on cardiovascular disease, however, are controversial; many studies have reported negative effects, others have reported no effects. ${ }^{6}$ Many studies have examined whether coffee intake is involved in the elevation of blood pressure, ${ }^{7,8}$ but to date there is no evidence for its direct involvement. ${ }^{9,10}$ Funatsu et al. ${ }^{11}$ recently reported that daily ingestion of brewed coffee from roasted coffee beans reduces blood pressure in humans who habitually drink alcoholic beverages. Recent animal and human studies reported an antihypertensive effect of chlorogenic acids in green coffee bean extract. $^{12,13}$ The relationship between the ingestion and the antihypertensive effect of brewed coffee from roasted coffee beans and blood pressure, however, is not yet clear.

Suzuki et al. ${ }^{14}$ recently reported that hydroxyhydroquinone (HHQ) produced by roasting green coffee beans inhibits the antihypertensive effect of chlorogenic acids in brewed coffee. Furthermore, an antihypertensive effect of brewed coffee was shown after reducing HHQ in an animal study using spontaneously hypertensive rats. HHQ produces reactive oxygen species (ROS), ${ }^{15}$ and ROS are assumed to reduce the antihypertensive effect of chlorogenic acids. Furthermore, a 12-week continuous ingestion period of chlorogenic acids contained in HHQ-reduced coffee decreases the blood pressure and improves the vascular endothelial function in mildly hypertensive subjects. ${ }^{16,17}$ The mechanism underlying this effect, however, remains unclear. Here, we investigated factors that may influence blood pressure when drinking HHQ-reduced coffee, in subjects with mild hypertension.

\section{METHODS}

Study subjects

The subjects were men and women aged 30-64 years at the start of the study who were not taking any antihypertensive drugs, had a moderate risk for mild hypertension (systolic blood pressure (SBP): 140 to $159 \mathrm{~mm} \mathrm{Hg}$, diastolic blood pressure (DBP): $99 \mathrm{~mm} \mathrm{Hg}$ or less), ${ }^{18}$ and had two or more risk factors for lifestyle-related diseases. The risk factors were as follows: waist circumference

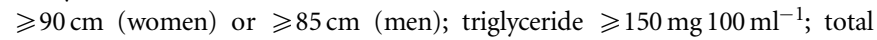

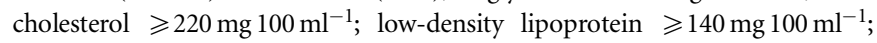

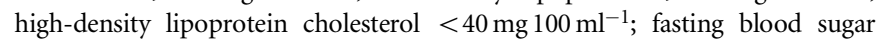

${ }^{1}$ Health Care Food Research Laboratories, Kao Corporation, Tokyo, Japan; ${ }^{2}$ Department of Geriatric Medicine, Osaka University Graduate School of Medicine, Osaka, Japan and ${ }^{3}$ Department of Health Promotion Science, Osaka University Graduate School of Medicine, Osaka, Japan

Correspondence: R Ochiai, Health Care Food Research Laboratories, Kao Corporation, 2-1-3, Bunka Sumida-ku, Tokyo 131-8501, Japan.

E-mail: ochiai.ryuujikao.co.jp

Received 18 May 2009; revised 17 June 2009; accepted 14 July 2009; published online 28 August 2009 
$\geqslant 110 \mathrm{mg} 100 \mathrm{ml}^{-1}$; heavy smoking (20 cigarettes per day or more). The vascular failure was diagnosed by SBP value corresponding to mild hypertension, an endothelium-dependent flow-mediated vasodilation (FMD) of $<10 \%$, and a ratio of FMD to endothelium-independent vasodilation with nitroglycerin (NTGD) of $<0.5$ during the run-in period were enrolled in the study. Subjects who met the following criteria were excluded: severe liver dysfunction, cardiovascular disorders, endocrine disorders, or caffeine/coffee hypersensitivity/allergy, pregnant women/those who may be pregnant or ineligibility as judged by attending physicians.

\section{Study procedures}

The study was approved by the Ethics Review Board of the Osaka Pharmacology Clinical Research Hospital: OPHAC Hospital. Written informed consent was obtained from all subjects after contents, features, purpose and expected risks of the study were explained using a document by the attending physicians. The study was conducted under the supervision of the chief investigator in accordance with the Declaration of Helsinki (2002), and followed the tenets of the Declaration of Helsinki.

\section{Test beverages}

Normal brewed coffee contains both chlorogenic acids and HHQ. On the basis of our high-performance liquid chromatography analysis, a cup of coffee typically contains $40-350 \mathrm{mg}$ of chlorogenic acids and $0.1-1.7 \mathrm{mg}$ HHQ. In preparing an active test beverage, we reduced the level of HHQ in the coffee by an adsorption treatment, and adjusted the chlorogenic acid content to $300 \mathrm{mg}$ per $184 \mathrm{ml}$. A placebo beverage was prepared by reducing the levels of chlorogenic acids and HHQ. Furthermore, commercially available coffee (control beverage) was given during the induction period before test beverage consumption, and during the follow-up period after the test beverage consumption. The beverage form was canned coffee $(184 \mathrm{ml})$, which is popular in Japan. We prepared these beverages so that there were no differences in their appearance or flavor. Caffeine, which may influence blood pressure, was present in the active, placebo and control beverages at 79,59 and $70 \mathrm{mg}$ per $184 \mathrm{ml}$, respectively. The caloric value of all beverages was $9 \mathrm{kcal}$ per $184 \mathrm{ml}$ (Table 1).

\section{Protocol}

The study design was a double blind, randomized, placebo-controlled intervention study, with a 4-week run-in period, an 8-week test beverage ingestion period, and a 2-week follow-up period (Figure 1). A screening test was

\section{Table 1 Compositions of test beverage}

\begin{tabular}{lccc}
\hline & Control beverage & Active beverage & Placebo beverage \\
\hline Chlorogenic acids (mg) & 134 & 300 & 0 \\
Hydroxyhydroquinone (mg) & 0.12 & 0.03 & 0.03 \\
Caffeine (mg) & 70 & 79 & 59 \\
Energy (kcal) & 9 & 9 & 9 \\
\hline
\end{tabular}

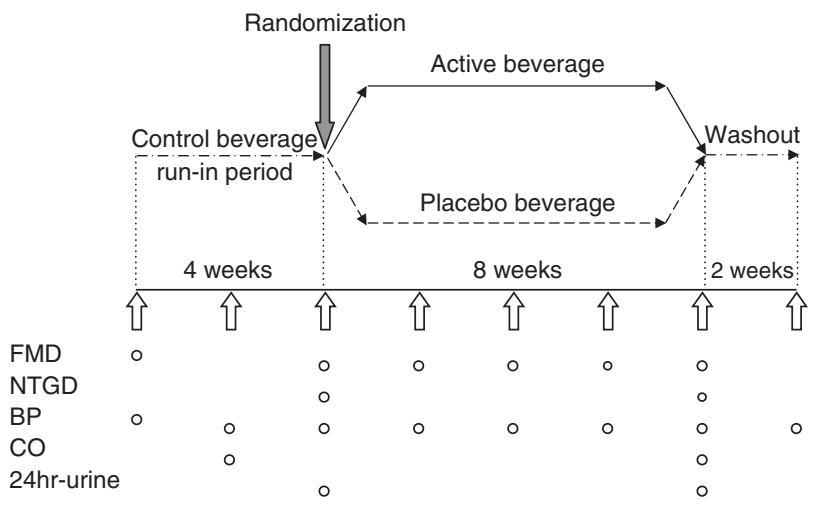

Figure 1 Study design. administered 4 weeks ( $-4 \mathrm{wk}$ ) before the start of test beverage ingestion, Week $0(0 \mathrm{wk})$. Subsequent screening tests were conducted 2 weeks before the start ( $-2 \mathrm{wk}$ ), at Weeks $0,2,4,6$ and 8 (0, 2, 4, 6 and 8 wk, respectively); and 2 weeks after the end of the test beverage ingestion $(+2 \mathrm{wk})$. After the screening test at $-2 \mathrm{wk}$, we divided the subjects into two groups using the minimization method, in which they were stratified with respect to FMD, blood pressure values classified in accordance with the JSH2004 guidelines, ${ }^{18}$ sex and age. The subjects consumed the active or placebo beverages ( 1 can per day) during the ingestion period. During the run-in and follow-up periods, all subjects consumed the control beverage ( 1 can per day). They were instructed to continue their usual eating habits while avoiding overeating/drinking during the study period, and not to change exercise/smoking habits. Furthermore, they were prohibited from taking agents that may influence blood pressure, foods that may reduce hypertension, and coffee other than the test beverages. On the day before examination, they ate an evening meal before 2100 hours, and were prohibited from eating/drinking anything other than water until the end of the examination. On the day of examination, they were prohibited from smoking until the end of the examination. During the consumption period, on examination days they were instructed to drink the test beverage after the end of the examination. Examination parameters included FMD, NTGD, cardiac output, SBP, the second peak of systolic blood pressure (SBP2), DBP, total peripheral resistance (TPR), pulse, height, body weight, hematologic/ biochemical parameters, urinalysis and interview.

\section{Physiologic parameters}

$F M D$. The subjects rested in a supine position for $20 \mathrm{~min}$ or more in an airconditioned room $\left(22 \pm 2{ }^{\circ} \mathrm{C}\right)$. A blood pressure cuff was placed on the forearm contralateral to their dominant hand, and the brachial artery (BA) diameter in the supra-elbow area of the upper arm was measured using ultrasonography (Nemio SSA-550A, TOSHIBA, Tokyo, Japan), and regarded as the resting BA diameter. The blood pressure cuff was then inflated to $200 \mathrm{~mm} \mathrm{Hg}$ for a period of $5 \mathrm{~min}$. The BA diameter was measured immediately after deflation of the cuff, and the FMD was calculated based on the maximum BA diameter measured during the 6-min period.

NTGD. After the FMD measurement, we confirmed that the BA diameter had returned to the pre-FMD value. The resting BA diameter was measured $10 \mathrm{~min}$ later. A total of $0.3 \mathrm{mg}$ nitroglycerin was then administered sublingually. The BA diameter was measured for $6 \mathrm{~min}$. The NTGD was calculated based on the maximum BA diameter.

Cardiac output. The subjects were rested in a left-sided position, and the time velocity integral and diameter of the left ventricular outflow tract were measured by ultrasonography. The cardiac output was calculated based on these values.

Blood pressure/TPR/pulse. After a more than 10-min rest in a sitting position, the SBP, SBP2, DBP and pulse were measured more than twice at 1- to 2-min intervals using an automatic blood pressure meter (HEM-9000AI, Omron Healthcare, Kyoto, Japan). The mean of two stable SBP values (difference between the two values: $\leqslant 5 \mathrm{~mm} \mathrm{Hg}$ ) was used. For SBP2, DBP and pulse rate, means were calculated from the readings selected for the determination of the mean SBP. TPR was calculated based on cardiac output, SBP and DBP.

FMD was measured at $-4,0,2,4,6$ and $8 \mathrm{wk}$, NTGD was measured at 0 and $8 \mathrm{wk}$, and cardiac output was measured at -2 and $8 \mathrm{wk}$. SBP, SBP2, DBP and pulse values were measured on all visit days.

Physical parameters, blood biochemistry, urinalysis and interview Height was measured only at -4 wk. Body weight, abdominal circumference and body temperature were measured at all visits. Hematology and urinalysis were carried out at 0 and $8 \mathrm{wk}$. The blood chemistry parameters, triglyceride, total cholesterol, low-density lipoprotein cholesterol, high-density lipoprotein cholesterol, fasting blood sugar, hemoglobin Alc, high-sensitive C-reactive protein, soluble inter-cellular adhesion molecule-1, homocysteine and glutathione were measured at the OPHAC Hospital or at SRL (Tokyo, Japan). Spot urinalysis and measurements of protein, $\mathrm{pH}$, urine sugar, urobilinogen, ketone 
bodies, occult blood, isoprostane and creatinine were carried out at the OPHAC Hospital or SRL. For 24-h urine, urine was collected using a proportional collector (Urine Mate P, Sumitomo Bakelite, Tokyo, Japan), and the urine volume and levels of sodium and potassium were measured at the OPHAC Hospital or at SRL. A physician interviewed the subjects to evaluate their subjective symptoms and objective findings.

\section{Statistical analysis}

The primary endpoint was to clarify the association between the blood pressure-reducing effects and vasoreactivity of HHQ-reduced coffee beverage in mildly hypertensive subjects.

A group with subjects who were considered to be eligible during the induction period and could comply with the protocol without dropping out of the study during the consumption period was named per protocol set. To evaluate variation in FMD, NTGD, SBP, SBP2 and DBP values, a linear mixedmodel repeated-measures analysis of covariance was used to assess the significance of changes in the actual value, the baseline values as covariates, and the measured values at 2, 4, 6 and $8 \mathrm{wk}$ as variables.

To evaluate cardiac output, TPR, pulse, body weight and urinalysis/hematologic data, a linear mixed-model repeated-measures analysis of covariance was used to assess the significance of changes in the actual value, the baseline values as covariates, and the measured values at $8 \mathrm{wk}$ as variables. For the comparison of subject background and hematologic data at the baseline, we used Student's $t$-test. Linear data are expressed as mean \pm s.e. All tests were paired. A $P$-value $<0.05$ was regarded as significant. Statistical calculations were carried out using SPSS 15.0J software for Windows (SPSS Japan, Tokyo, Japan).

\section{RESULTS}

\section{Subjects}

Participant flow during the study period is shown in Figure 2. In this study, subjects were invited to participate over a period of 6 months, and subjects who were considered to be eligible during the induction period were assigned to the active or placebo groups through stratified randomization. A total of 58 subjects underwent screening; 19 subjects were excluded because they did not meet eligibility criteria, and 39 subjects were judged to be eligible at the run-in period and were randomized to the active and placebo groups. After being placed in the beverage groups, the following subjects were excluded: 12 subjects who did not have mild hypertensive blood pressure at $0 \mathrm{wk}$, and 4 subjects who had normal endothelial function at $0 \mathrm{wk}$. Out of the subjects who completed the study, the following were excluded: 1 subject failed NTGD measurement, and 1 subject violated the protocol. A total of 21 subjects were analyzed for the primary endpoint: 9 in the active beverage group and 12 in the placebo beverage group. The baseline characteristics of the groups are shown in Tables $2-4$. There were no significant differences in the baseline values for any parameter other than the blood total glutathione level (Table 4) between the active and placebo beverage groups. The consumption rates for the beverages in the two groups were $99 \%$ or more, with no significant difference between groups. Changes in the SBP, SBP2, DBP, FMD and NTGD values are shown in Table 2 . The baseline body weight, body mass index, heart rate and cardiac output values are shown in Table 3.

\section{Blood pressure}

The SBP and SBP2 decreased significantly from their baseline values after 6 and $8 \mathrm{wk}$ in the active group. In the placebo group, SBP decreased significantly from baseline values after 2 and $8 \mathrm{wk}$, the SBP2 decreased significantly from baseline at only $2 \mathrm{wk}$. However, there was no significant difference at each time point between the groups. There was no significant difference in DBP value.

\section{FMD}

The FMD value increased significantly from baseline value after $2 \mathrm{wk}$ in the active group, but in the placebo group there was no significant change from the baseline value. There was a significant group-by-time interaction for FMD. There were no significant changes in the NTGD value. In addition, no significant differences between the groups were noted in the cardiac output, TPR, pulse, body weight and body mass index.

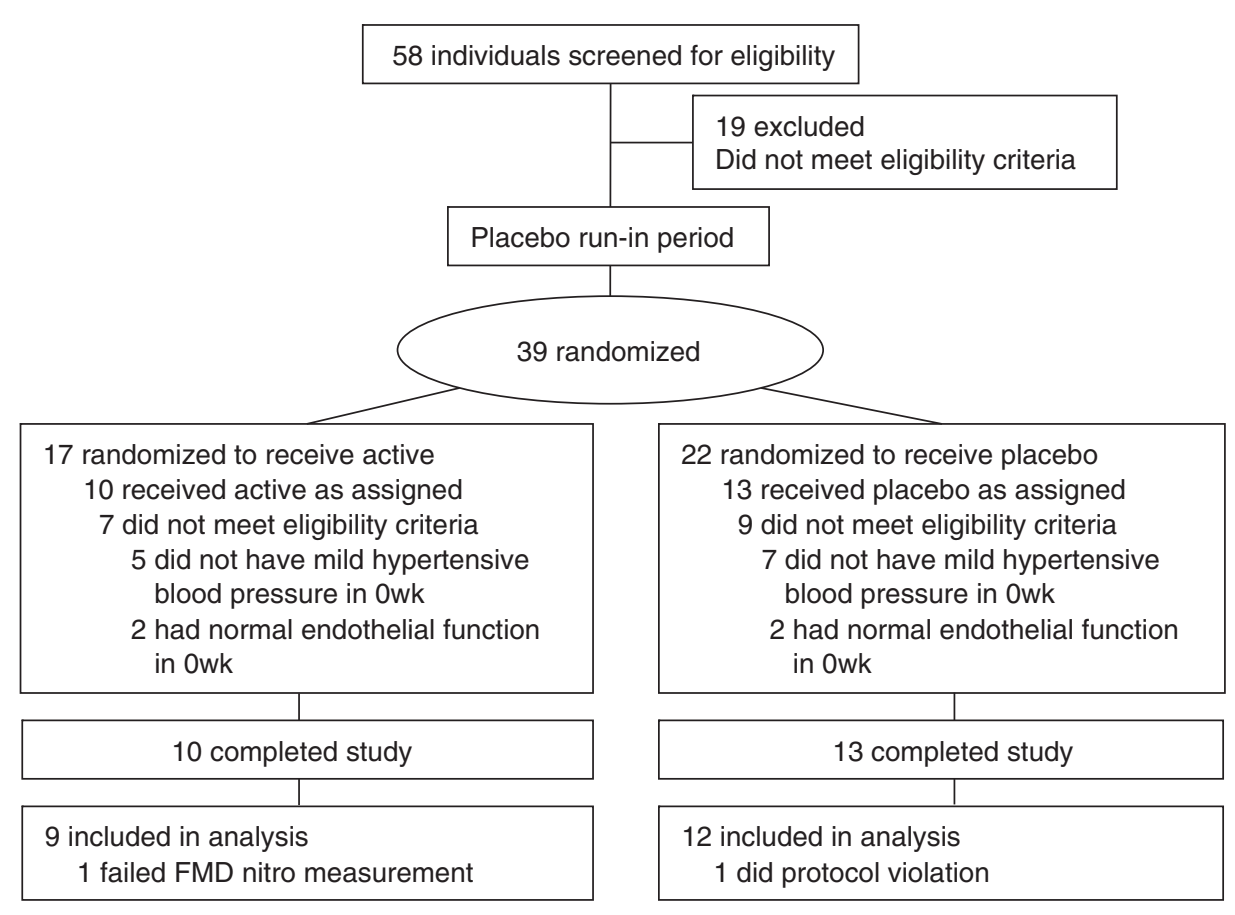

Figure 2 Participants flow. 
Table 2 Change in blood pressure, FMD and NTGD

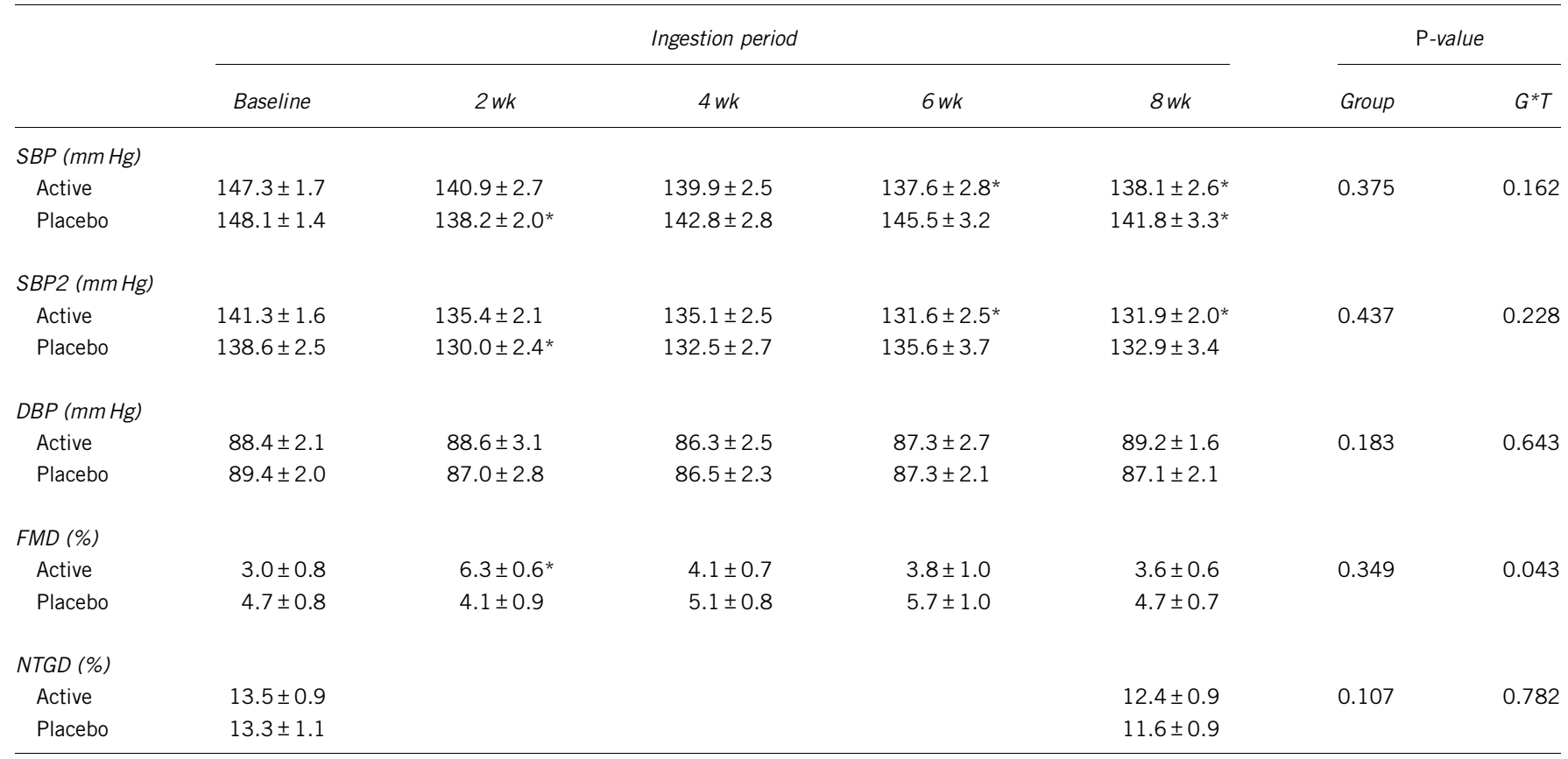

Abbreviations: DBP, diastolic blood pressure; FMD, endothelium-dependent flow-mediated vasodilation; NTGD, endothelium-independent vasodilation with nitroglycerin; SBP, systolic blood pressure; SBP, second peak of systolic blood pressure.

Group presents $P$-value of group effect by repeated measures analysis of covariance (covariate=baseline, variable $=2,4,6$ and 8 wk)

G*T represents $P$-value of group $*$ week effect by repeated measures analysis of covariance (covariate $=$ baseline, variable $=2,4,6$ and 8 wk).

*Represents $P<0.05$; change from baseline.

Table 3 Change in weight, pulse, CO, TPR and 24-h urinary excretion

\begin{tabular}{|c|c|c|c|c|c|c|}
\hline & \multicolumn{2}{|c|}{ Active } & \multicolumn{2}{|c|}{ Placebo } & \multicolumn{2}{|c|}{$\mathrm{P}$-value } \\
\hline & Baseline & $8 w k$ & Baseline & $8 w k$ & Group & $G * T$ \\
\hline Weight (kg) & $68.2 \pm 3.6$ & $67.7 \pm 3.5$ & $64.6 \pm 3.5$ & $64.7 \pm 3.6$ & 0.873 & 0.718 \\
\hline $\mathrm{BMI}\left(\mathrm{kg} \mathrm{m}^{-2}\right)$ & $24.2 \pm 0.9$ & $24.0 \pm 0.9$ & $24.2 \pm 1.1$ & $24.2 \pm 1.2$ & 0.953 & 0.730 \\
\hline Pulse (beats per min) & $63.9 \pm 2.9$ & $63.7 \pm 2.2$ & $69.8 \pm 2.7$ & $70.2 \pm 2.9$ & 0.664 & 0.829 \\
\hline CO (I per min) & $4.3 \pm 0.3$ & $4.4 \pm 0.3$ & $5.4 \pm 0.5$ & $5.0 \pm 0.4$ & 0.147 & 0.158 \\
\hline TPR (dyne.s per $\mathrm{cm}^{5}$ ) & $2089 \pm 130$ & $1965 \pm 116$ & $1757 \pm 147$ & $1833 \pm 156$ & 0.245 & 0.174 \\
\hline Urinary excretion (I)a & $1.2 \pm 0.2$ & $1.1 \pm 0.1$ & $1.2 \pm 0.1$ & $1.4 \pm 0.2$ & 0.349 & 0.263 \\
\hline Urinary $\mathrm{Na}$ excretion (g per day) ${ }^{\mathrm{a}}$ & $3.7 \pm 0.2$ & $3.9 \pm 0.4$ & $3.4 \pm 0.5$ & $3.3 \pm 0.4$ & 0.350 & 0.590 \\
\hline Urinary $\mathrm{K}$ excretion (g per day) & $1.2 \pm 0.1$ & $1.7 \pm 0.3$ & $1.3 \pm 0.2$ & $1.4 \pm 0.2$ & 0.753 & 0.087 \\
\hline
\end{tabular}

Abbreviations: $\mathrm{CO}$, cardiac output; TPR, total peripheral resistance.

a24-h home urine collection.

Group presents $P$-value of group effect by repeated measures analysis of covariance (covariate=baseline, variable $=8 \mathrm{wk}$ ).

$\mathrm{G}^{*} \mathrm{~T}$ presents $P$-value of group ${ }^{*}$ week effect by repeated measures analysis of covariance (covariate=baseline, variable $=8 \mathrm{wk}$ ).

\section{Blood biochemistry and urinalysis}

Tables 3 and 4 show results for blood biochemistry and urinalysis. In the active beverage group, the blood total glutathione level was significantly increased after $8 \mathrm{wk}$, and the urinary isoprostane level was significantly decreased. In the placebo group, no significant change from baseline was noted. No significant differences were noted between the groups for the 24-h urine volume and urinary sodium/potassium excretions, and qualitative urinalysis (data not shown).

\section{Adverse events}

No adverse events related to the ingestion of HHQ-reduced coffee were noted in the interviews taken by physician. There were no clinically problematic changes in laboratory test values for any subject.

\section{DISCUSSION}

In this study, the subjects that ingested the HHQ-reduced, high chlorogenic acid-containing coffee (the active beverage group) showed a significant decrease in SBP from the baseline after 6 weeks or more, thereby confirming previous findings regarding the antihypertensive effect of HHQ-reduced coffee. In addition, there was a significant decrease in the SBP2, an index of central aortic systolic blood pressure, ${ }^{19,20}$ after 6 weeks of test beverage ingestion. Therefore, the active beverage may have antihypertensive effects through its actions on the arteries. As HHQ-reduced coffee ingestion reduced both peripheral and central blood pressures, it may be useful for preventing cardiovascular disorders, such as myocardial infarction and arteriosclerosis. ${ }^{20,21}$ In the placebo beverage group, SBP decreased significantly from baseline values after 2 and 8 weeks. SBP2 similarly 
Table 4 Change in urinalysis and blood chemistry parameters

\begin{tabular}{|c|c|c|c|c|c|}
\hline & \multicolumn{2}{|c|}{ Active } & \multicolumn{2}{|c|}{ Placebo } & \multirow{2}{*}{$\begin{array}{c}\text { P-value } \\
\text { Group }\end{array}$} \\
\hline & Baseline & $8 w k$ & Baseline & $8 w k$ & \\
\hline $\mathrm{HbA}_{1 \mathrm{C}}(\%)$ & $5.3 \pm 0.1$ & $5.4 \pm 0.1$ & $5.2 \pm 0.1$ & $5.2 \pm 0.1$ & 0.102 \\
\hline Triglyceride (mg $100 \mathrm{ml}^{-1}$ ) & $118.9 \pm 13.4$ & $150.7 \pm 16.2$ & $110.3 \pm 16.1$ & $146.7 \pm 28.6$ & 0.815 \\
\hline Total cholesterol (mg $100 \mathrm{ml}^{-1}$ ) & $214.3 \pm 12.5$ & $222.6 \pm 11.4$ & $218.4 \pm 14.7$ & $219.7 \pm 13.2$ & 0.975 \\
\hline Homocysteine $\left(\mathrm{nmol} \mathrm{ml} \mathrm{l}^{-1}\right)$ & $11.0 \pm 1.1$ & $10.7 \pm 0.8$ & $17.8 \pm 6.4$ & $20.3 \pm 7.6$ & 0.325 \\
\hline $\operatorname{hsCRP}\left(\mathrm{ng} \mathrm{ml}^{-1}\right)$ & $794.5 \pm 258.1$ & $836.6 \pm 289.7$ & $656.2 \pm 147.6$ & $629.4 \pm 199.5$ & 0.643 \\
\hline sICAM-1 (ng ml-1) & $159.7 \pm 14.9$ & $172.6 \pm 19.3$ & $209.8 \pm 22.6$ & $216.4 \pm 25.5$ & 0.149 \\
\hline Total glutathione $\left(\mathrm{mg} \mathrm{ml}^{-1}\right)$ & $32.4 \pm 3.2$ & $39.2 \pm 2.5^{*}$ & $40.4 \pm 2.2$ & $41.7 \pm 1.3$ & 0.088 \\
\hline urinary isoprostane (pg per ml per cre) & $151.6 \pm 22.1$ & $121.0 \pm 19.7^{*}$ & $217.6 \pm 25.4$ & $186.9 \pm 18.4$ & 0.037 \\
\hline
\end{tabular}

Abbreviations: HDL, high-density lipoprotein; LDL, low-density lipoprotein; hsCRP, high sensitivity C-reactive protein; sICAM-1, soluble intercellular adhesion molecule-1. Group presents $P$-value of group effect by repeated measures analysis of covariance (covariate=baseline, variable $=8 \mathrm{wk}$ ).

*Represents $P<0.05$; change from baseline.

decreased after 2 weeks. This suggests that the placebo beverage did not act on the arteries; the decrease in SBP2 at 2 weeks might indicate a placebo effect related to the start of test beverage ingestion. There was no significant difference between the groups with regard to blood pressure. It was suggested that the reasons for the unexpected results were as follows: the sample size was small; placebo effects were expressed.

Factors influencing the blood pressure-decreasing effects, such as the FMD, NTGD, cardiac output, TPR, urine volume and body weight, are shown in Tables 2-4. The FMD value in the active beverage group increased significantly from baseline values after 2 weeks (Table 2); this change was noted before $6 \mathrm{wk}$, at which time there was a decrease in the blood pressure. In the placebo beverage group, there were no significant changes.

On the basis of these findings, the amelioration of vasoreactivity may be involved in the antihypertensive effect of an HHQ-reduced coffee beverage containing $300 \mathrm{mg}$ of chlorogenic acids. In this study, there were no changes in the NTGD after continuous consumption, suggesting a vascular endothelium-derived amelioration of vasoreactivity.

The hematology and urinalysis results at baseline and after 8 weeks of test beverage ingestion (Tables 3 and 4) showed that there were differences in the pre- and post-ingestion urinary isoprostane between the active and placebo beverage groups. In addition, after active beverage ingestion, there was a significant increase in the blood level of glutathione, an enzyme that removes ROS in vivo. The placebo beverage group did not show any similar changes. Levels of oxidative stress markers, such as 8-isoprostane, increase with an elevation in blood pressure. ${ }^{22,23}$ Reducing ROS might be involved in the antihypertensive action mechanism of the active beverage.

Several studies have reported an antihypertensive effect of HHQreduced coffee in subjects with mild hypertension or high-normal blood pressure. ${ }^{15,16}$ Chlorogenic acids may contribute to these antihypertensive effects. Suzuki et al. ${ }^{24}$ conducted an experiment in spontaneously hyperactive rats, and hypothesized that the mechanism underlying the hypotensive action of chlorogenic acids is as follows: the ingestion of chlorogenic acids may inhibit the excessive synthesis of ROS, decreasing the blood pressure via improvement in NO availability in vivo and in vascular endothelial function. This hypothesis is supported by results of this study. Another study in humans also indicated that consumption of a green coffee bean extract (main component: chlorogenic acids) enhances vascular endothelial function. ${ }^{25}$ These results indicate that ingestion of HHQ-reduced coffee decreased blood pressure in subjects with mild hypertension. The decreased blood pressure was associated with reduced ROS and improved vascular endothelial function.

\section{Study limitations}

There were several limitations to our study. First, the sample size was relatively small. For an accurate evaluation of the vascular endothelial function based on FMD, the study must be conducted in a larger number of subjects. Second, the subjects comprised mildly hypertensive patients with vascular failure; the results are not applicable to all patients with hypertension. Future studies involving a larger number of patients with mild hypertension should be carried out to address these issues.

\section{ACKNOWLEDGEMENTS}

We thank the subjects and physicians/health professionals who participated in this study for their cooperation.

1 van Dam RM, Feskens EJ. Coffee consumption and risk of type 2 diabetes mellitus. Lancet 2002; 360: 1477-1478.

2 Salazar-Martinez E, Willett WC, Ascherio A, Manson JE, Leitzmann MF, Stampfer MJ, Hu FB. Coffee consumption and risk for type 2 diabetes mellitus. Ann Intern Med 2004; 140: 1-8.

3 Nilssen O, Forde $\mathrm{OH}$, Brenn T, The Tromso Study. Distribution and population determinants of gamma-glutamyltransferase. Am J Epidemiol 1990; 132: 318-326.

4 Kono S, Shinchi K, Imanishi K, Todoroki I, Hatsuse K. Coffee and serum gammaglutamyltransferase: a study of self-defense officials in Japan. Am J Epidemiol 1994; 139: 723-727.

5 Hernan MA, Takkouche B, Caamano-Isorna F, Gestal-Otero JJ. A meta-analysis of coffee drinking, cigarette smoking, and the risk of Parkinson's disease. Ann Neurol 2002; 52: 276-284.

6 Lopez-Garcia E, van Dam RM, Willett WC, Rimm EB, Manson JE, Stampfer MJ, Rexrode $\mathrm{KM}$, Hu FB. Coffee consumption and coronary heart disease in men and women: a prospective cohort study. Circulation 2006; 113: 2045-2053.

7 Jee SH, He J, Whelton PK, Suh I, Klag MJ. The effect of chronic coffee drinking on blood pressure: a meta-analysis of controlled clinical trials. Hypertension 1999; 33 : 647-652.

8 Nurminen ML, Niittynen L, Korpela R, Vapaatalo H. Coffee, caffeine and blood pressure: a critical review. Eur J Clin Nutr 1999; 53: 831-839.

9 Wilson PW, Garrison RJ, Kannel WB, McGee DL, Castelli WP. Is coffee consumption a contributor to cardiovascular disease? Insights from the Framingham Study. Arch Intern Med 1989; 149: 1169-1172.

10 MacDonald TM, Sharpe K, Fowler G, Lyons D, Freestone S, Lovell HG, Webster J, Petrie JC. Caffeine restriction: effect on mild hypertension. BMJ 1991; 303: 1235-1238. 
11 Funatsu K, Yamashita T, Nakamura H. Effect of coffee intake on blood pressure in male habitual alcohol drinkers. Hypertens Res 2005; 28: 521-527.

12 Suzuki A, Kagawa D, Ochiai R, Tokimitsu I, Saito I. Green coffee bean extract and its metabolites have a hypotensive effect in spontaneously hypertensive rats. Hypertens Res 2002; 25: 99-107.

13 Watanabe T, Arai Y, Mitsui Y, Kusaura T, Okawa W, Kajihara Y, Saito I. The blood pressure-lowering effect and safety of chlorogenic acid from green coffee bean extract in essential hypertension. Clin Exp Hypertens 2006; 28: 439-449.

14 Suzuki A, Fujii A, Yamamoto M, Yamamoto M, Ohminami H, Kameyama A, Shibuya Y, Nishizawa Y, Tokimitsu I, Saito I. Improvement of hypertension and vascular dysfunction by hydroxyhydroquinone-free coffee in a genetic model of hypertension. FEBS Lett 2006; 580: 2317-2322.

15 Halliwell B, Long L, Yee T, Lim S, Kelly R. Establishing biomarkers of oxidative stress: the measurement of hydrogen peroxide in human urine. Curr Med Chem 2004; 11 : 1085-1092.

16 Chikama A, Yamaguchi T, Watanabe T, Mori K, Katsuragi Y, Tokimitsu I, Kajimoto O, Kitakaze M. Effects of chlorogenic acids in hydroxyhydroquinone-reduced coffee on blood pressure and vascular endothelial function in humans. Prog Med 2006; 26 : 1723-1736.

17 Nagao T, Ochiai R, Katsuragi Y, Hayakawa Y, Kataoka K, Komikado M, Tokimitsu I, Tsuchida T. Hydroxyhydroquinone-reduced milk coffee decreases blood pressure in individuals with mild hypertension and high-normal blood pressure. Prog Med 2007; 27: 2649-2664
18 The Japanese Society of Hypertension. Guidelines for the Management of Hypertension (JSH2004). The Japanese Society of Hypertension: Tokyo, 2004.

19 Pauca AL, Kon ND, O'rourke MF. The second peak of the radial artery pressure wave represents aortic systolic pressure in hypertensive and elderly patients. $\mathrm{Br} \mathrm{J}$ Anaesth 2004; 92: 651-657.

20 Takazawa K, Kobayashi H, Shindo N, Tanaka N, Yamashina A. Relationship between radial and central arterial pulse wave and evaluation of central aortic pressure using the radial arterial pulse wave. Hypertens Res 2007; 30: 219-228.

21 Williams B, Lacy PS, Thom SM, Cruickshank K, Stanton A, Collier D, Hughes AD, Thurston $\mathrm{H}$, O'Rourke M. Differential impact of blood pressure-lowering drugs on central aortic pressure and clinical outcomes. Principal results of the Conduit Artery Function Evaluation (CAFE) Study. Circulation 2006; 113: 1213-1225.

22 Hozawa A, Ebihara S, Ohmori K, Kuriyama S, Ugajin T, Koizumi Y, Suzuki Y, Matsui T, Arai H, Tsubono Y, Sasaki H, Tsuji I. Increased plasma 8-isoprostane levels in hypertensive subjects: the Tsurugaya Project. Hypertens Res 2004; 27: 557-561.

23 Rodrigo R, Prat H, Passalacqua W, Araya J, Guichard C, Bächler JP. Relationship between oxidative stress and essential hypertension. Hypertens Res 2007; 30: 1159-1167.

24 Suzuki A, Yamamoto N, Jokura H, Yamamoto M, Fujii A, Tokimitsu I, Saito I. Chlorogenic acid attenuates hypertension and improves endothelial function in spontaneously hypertensive rats. J Hypertens 2006; 24: 1065-1073.

25 Ochiai R, Jokura H, Suzuki A, Tokimitsu I, Ohishi M, Komai N, Rakugi H, Ogihara T. Green coffee bean extract improves human vasoreactivity. Hypertens Res 2004; 27: 731-737. 